\title{
CARACTERIZAÇÃO DE UMA FLORESTA ESTACIONAL SEMIDECIDUAL EM VARGINHA, MG. E COMPARAÇÃO COM REMANESCENTES DA REGIÃO
}

\author{
Rafaela Pereira Naves ${ }^{1}$, Eduardo van den Berg ${ }^{2}$
}

(recebido: 6 de maio de 2010; aceito: 28 de fevereiro de 2012)

RESUMO: Foi realizado o levantamento florístico e fitossociológico na porção mais extensa de mata no Parque Florestal Municipal São Francisco de Assis, na cidade de Varginha, sul de Minas Gerais. A área tem cerca de 10 ha e é cortada por um pequeno córrego. Foram lançadas 25 parcelas de $20 \mathrm{~m}$ x $20 \mathrm{~m}$ e medidos e cadastrados todos os indivíduos arbóreos com DAP $\geq 5 \mathrm{~cm}$. Foram encontrados 1568 indivíduos que, juntos, somaram área basal de 23,65 m², sendo registrados 102 espécies, 72 gêneros e 42 famílias no levantamento fitossociológico e 111 espécies, 77 gêneros e 43 famílias no levantamento florístico. As famílias com maior número de espécies foram Myrtaceae, Fabaceae e Melastomataceae. As espécies de maior valor de importância foram Casearia arborea, Copaifera langsdorffii, Tachigali rugosa e Myrcia splendens. Os resultados encontrados são os esperados para as Florestas Estacionais Semideciduais da região.

Palavras-chave: Estrutura, diversidade, comunidades arbóreas.

\section{CHARACTERIZATION OF A SEMIDECIDUOUS FOREST IN VARGINHA, MG. AND COMPARISON WITH REMAINING FOREST FRAGMENTS IN THE REGION}

\begin{abstract}
A floristic and phytossociological survey was made at the biggest area of continue forest at Parque Florestal Municipal São Francisco de Assis, in Varginha county, south of Minas Gerais state. The area has 10 ha and possesses a water stream. It was sampled 25 plots of $20 \mathrm{~m} \times 20 \mathrm{~m}$ and recorded all the individuals with diameter at breast height (DBH) equal to or larger than $5 \mathrm{~cm}$. The phytossociological survey recorded 1568 individuals, and basal area of $23.65 \mathrm{~m}^{2}$.ha $\mathrm{h}^{-1}$ distributed in 103 species, 72 genera and 42 families. The floristic survey recorded 111 species, 77 genera and 43 families. The families with bigger number of species were: Myrtaceae, Fabaceae and Melastomataceae and the most important species were Casearia arborea, Copaifera langsdorffi, Tachigali rugosa and Myrcia splendens. The obtained results are in accordance wih the expected ones for the semideciduous seasonal forests of the region.
\end{abstract}

Key words: Structure, diversity, tree community.

\section{INTRODUÇÃO}

O Brasil é um dos países megadiversos no mundo (MITTERMEIER, 1997). Dentro desse contexto de elevada diversidade, insere-se o Domínio Atlântico. Esse domínio é apontado como um dos mais prioritários no sentido do estabelecimento de estratégias e políticas conservacionistas, em razão da sua perda de área (resta cerca de $12 \%$ da cobertura original) e elevada diversidade biológica (RIBEIRO et al., 2009).

A vegetação primitiva do centro-sul do estado de Minas Gerais compreendia um complicado mosaico, composto por manchas de florestas, cerrado, campo limpo de altitude e campo rupestre (EITEN, 1982), resultando em uma elevada diversidade biológica regional. Essa considerável variação é decorrente da elevada heterogeneidade ambiental e da apresentação da região como uma transição entre os cerrados do Brasil Central e as Florestas Semidecíduas do Domínio Atlântico (OLIVEIRA-FILHO et al., 1994b).

$\mathrm{Na}$ referida região, as Florestas Estacionais Semideciduais foram criticamente reduzidas a pequenos remanescentes isolados e inseridos em uma matriz adversa (OLIVEIRA-FILHO et al., 1994a). O estudo da comunidade arbórea desses fragmentos teve início há cerca de 25 anos, com pesquisadores da Universidade Federal de Lavras, e tem resultado em um importante acúmulo de informações e conhecimento sobre a composição, estrutura e ecologia desses remanescentes (PEREIRA, 2003).

A cidade de Varginha, situada no sul do Estado, possui uma unidade de conservação localizada em seu perímetro urbano, constituída por vegetação aberta (cerrado) e florestal (Floresta Estacional Semidecidual),

\footnotetext{
${ }^{1}$ Bióloga, Mestranda em Recursos Florestais - Universidade de São Paulo - Escola Superior de Agricultura Luiz de Queiroz. - Av. Pádua Dias, n 11 Cx. P. 09 - 13418-900 - Piracicaba, SP, Brasil - rafaela_ufla@yahoo.com.br

${ }^{2}$ Agrônomo, Professor Doutor em Biologia Vegetal - Universidade Federal de Lavras - Departamento de Biologia - Cx. P. 3037 - $37200-000$ Lavras, MG, Brasil - eduardo.lavras@gmail.com
}

Cerne, Lavras, v. 18, n. 3, p. 361-370, jul./set. 2012 
apresentando nascentes no seu interior. Entretanto, a área em questão carece de estudos sobre a composição florística e o estado de conservação em que se encontra, mesmo porque não existem estudos sobre esse tipo de vegetação no município de Varginha.

Diante do apresentado, neste trabalho, objetivouse a caracterização da área de floresta a partir do estabelecimento de parcelas permanentes para a compilação de informações sobre a composição florística, a estrutura e a diversidade da comunidade arbórea, comparando-a floristicamente com outros estudos dessa natureza feitos na região, que está inserida na Bacia do Alto Rio Grande.

\section{MATERIAL E MÉTODOS}

\section{1 Área de estudo}

O estudo foi conduzido no Parque Florestal Municipal São Francisco de Assis, referido ao longo do texto como Parque São Francisco, na cidade de Varginha, no Sul de Minas Gerais, Brasil, cujas coordenadas centrais são $21^{\circ} 34^{\prime} 25 \mathrm{~S}$ e $45^{\circ} 24^{\prime} 2 \mathrm{O}$.

A altitude média da cidade é de $950 \mathrm{~m}$. O município encontra-se na Bacia do Alto Rio Grande $-21^{\circ} 00^{\prime}$ a $22^{\circ} 20^{\prime} \mathrm{S}$ e $43^{\circ} 40^{\prime}$ a $45^{\circ} 30^{\prime} \mathrm{W}$ - (GONZAGA et al., 2008), subbacia do Rio Verde. O clima, segundo Köppen, é Cwb, com inverno seco e com verões chuvosos e brandos. A temperatura média anual é de $22^{\circ} \mathrm{C}$, o período de chuva se estende de modo geral, de outubro a março (OLIVEIRA; MARTINS, 1986).

A área é protegida desde 1957 e foi elevada a categoria de Parque Florestal Municipal em 1982. Possui 110 ha cercados com arame, distribuídos em áreas de cerrado e de floresta (OLIVEIRA; MARTINS, 1986). O foco deste trabalho é a maior porção de mata, que se encontra na parte sudeste da área, sendo classificada como Floresta Estacional Semidecidual Montana por Veloso et al. (1991). O local possui cerca de 10 ha e é cortado por um pequeno córrego, que é alimentado por várias nascentes (Figura 1 a).

Embora distúrbios antrópicos, como a extração de madeira e o pastoreio pararam aproximadamente 50 anos atrás (comunicação pessoal de moradores antigos do entorno e funcionários do Parque São Francisco), diversas trilhas estão presentes na área, agora utilizadas pelo público em caminhadas ecológicas. Incêndios frequentemente são registrados no parque. Até o momento do estudo, o último incêndio grave foi documentado em 2007, não afetando a área estudada especificamente.

Cerne, Lavras, v. 18, n. 3, p. 361-370, jul./set. 2012

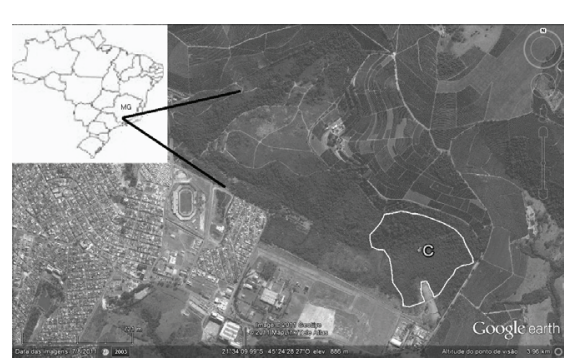

(a)

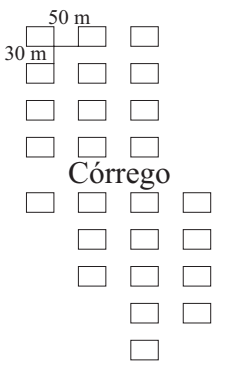

(b)
Figura 1 - (a) Imagem aérea do Parque Florestal Municipal São Francisco de Assis, Varginha, Minas Gerais, Brasil. A letra C indica o local onde foram alocadas as parcelas. Imagem Google Earth 2011. (b) Distribuição das 25 parcelas na área de estudo.

Figure 1 - (a) Aerial image of Parque Florestal Municipal São Francisco de Assis, Varginha, Minas Gerais, Brazil. The letter $C$ indicates where the plots were allocated. Image Google Earth 2011. (b) 25 plots distribution in the study area.

\subsection{Coleta e análise dos dados}

Foram alocadas sistematicamente 25 parcelas de $20 \mathrm{~m} \times 20 \mathrm{~m}$, ao longo de 4 transectos que cortaram o córrego perpendicularmente, totalizando 1 ha de amostragem. A distância entre os transectos foi de $50 \mathrm{~m} \mathrm{e}$ entre as parcelas $30 \mathrm{~m}$ (Figura $1 \mathrm{~b}$ ).

No levantamento estrutural, foram registrados todos os indivíduos arbóreos que apresentaram circunferência a altura do peito (1,30 $\mathrm{m}$ do solo) igual ou superior a 15,67 $\mathrm{cm}$ (que corresponde a $\mathrm{DAP} \geq 5 \mathrm{~cm}$ ). Para indivíduos que apresentaram bifurcação antes de $1,30 \mathrm{~m}$ de altura foi considerada a raiz quadrada da soma dos quadrados de todos os fustes, desde que a área basal de seus caules correspondesse àquela de um caule com $\mathrm{DAP} \geq 5 \mathrm{~cm}$ ( $\mathrm{SILVA}$ et al., 2003).

Todos os indivíduos amostrados dentro das parcelas receberam uma plaqueta de alumínio com número de identificação. Foram também coletadas espécies fora da parcela para compor o levantamento florístico.

As espécies foram identificadas por meio de comparações com o acervo do Herbário ESAL, da Universidade Federal de Lavras, por consulta à literatura pertinente e a especialistas. As espécies foram distribuídas em táxons, segundo APG III (2009). Todo o material botânico coletado foi depositado no Herbário ESAL.

\subsection{Estrutura e diversidade da comunidade}

Para caracterizar a estrutura da comunidade foram utilizados os parâmetros fitossociológicos de frequência, 
densidade e dominância absolutas e relativas. A partir destes foram obtidos os valores de importância para cada espécie (MUELLER-DOMBOIS; ELLENBERG, 1974).

Foram também obtidos os índices de diversidade de Shannon-Wiener e a equabilidade de Pielou (BROWER; ZAR, 1984).

\subsection{Comparação com outras áreas}

Para contextualizar a área de estudo em relação a outros remanescentes florestais da região, foram feitas comparações com as informações existentes em Pereira (2003), sobre 20 áreas de florestas semideciduais na região do Alto Rio Grande, que utilizaram o mesmo critério de inclusão (DAP $\geq 5 \mathrm{~cm}$ ) e amostraram cerca de 1 ha (Tabela 1). A classificação de Veloso et al. (1991) para essas áreas é Floresta Estacional Semidecidual Montana. Enquanto Oliveira-Filho e Fontes (2000) dividem as florestas de altitudes elevadas $(>700 \mathrm{~m})$ em dois pisos: inferomontanas $(>700 \mathrm{~m}-1100 \mathrm{~m})$ e altomontanas $(>1100 \mathrm{~m})$, portanto, quatro das áreas $(\mathrm{CB}$, IB, PB e PI) estariam nessa classificação das florestas de maiores altitudes.

Foi preparada uma matriz contendo dados de presença/ausência das espécies por área. A partir dessa matriz, fez-se a ordenação das áreas, por meio de uma análise de correspondência retificada (DCA), do inglês Detrened Correspondence Analysis (KENT; COKER, 1992) para comparar o Parque São Francisco com essas outras 20 áreas, utilizando o Programa PC-Ord for Windows versão 5.0 (MCCUNE; MEFFORD, 2006).

Tabela 1 - Informações geográficas das 21 áreas de florestas da região do Alto Rio Grande. $\mathrm{S}=$ riqueza, $\mathrm{AB}=$ área basal, $\mathrm{DA}=$ densidade absoluta, $\mathrm{H}$ = índice de diversidade de Shannon, $\mathrm{J}=$ equabilidade de Pielou. Tabela adaptada a partir de Pereira (2003).

Table 1 - Geographical information of the 21 forest areas of the Alto Rio Grande region. $S=$ number of species, AB = basal area, $D A=$ absolute dominancy, $H=$ Shannon's index, $J=$ Pielou's evenness index. Table adapted from Pereira (2003).

\begin{tabular}{|c|c|c|c|c|c|c|c|c|c|c|c|}
\hline $\begin{array}{l}\text { Nome } \\
\text { abreviado }\end{array}$ & Local & $\begin{array}{l}\text { Área } \\
\text { (ha) }\end{array}$ & Latitude & Longitude & $\begin{array}{l}\text { Altitude } \\
\text { (m) }\end{array}$ & Clima & $\mathrm{S}$ & $\begin{array}{c}\mathrm{AB} \\
\left(\mathrm{m}^{2} \cdot \mathrm{ha}^{-1}\right)\end{array}$ & $\begin{array}{c}\text { DA } \\
\text { (ind.ha }{ }^{-1} \text { ) }\end{array}$ & $\mathrm{H}$ & $\mathrm{J}$ \\
\hline $\mathrm{BS}$ & Bom sucesso & 83,45 & $21^{\circ} 09^{\prime} 27^{\prime \prime}$ & $44^{\circ} 54^{\prime} 00^{\prime \prime}$ & 806 a 832 & $\mathrm{Cwb}$ & 137 & 34,99 & 2063 & 4,28 & 0,87 \\
\hline $\mathrm{CM}$ & Camargos & 10,36 & $21^{\circ} 21^{\prime} 18^{\prime \prime}$ & $44^{\circ} 36^{\prime} 38^{\prime \prime}$ & 913 a 960 & $\mathrm{Cwb}$ & 112 & 30,45 & 1936,9 & 3,74 & 0,79 \\
\hline $\mathrm{CP}$ & Capivari & 9,78 & $21^{\circ} 16^{\prime} 23^{\prime \prime}$ & $44^{\circ} 52^{\prime} 53^{\prime \prime}$ & 825 a 875 & $\mathrm{Cwb}$ & 125 & 27,7 & 1487,5 & 4,21 & 0,87 \\
\hline $\mathrm{CR}$ & Carrancas & 35,98 & $21^{\circ} 36^{\prime} 29^{\prime \prime}$ & $44^{\circ} 36^{\prime} 38^{\prime \prime}$ & 1.440 a 1.513 & Cwa & 105 & 34,16 & 2137,5 & 3,89 & 0,84 \\
\hline IB & Ibitipoca & 95,05 & $21^{\circ} 42^{\prime} 13^{\prime \prime}$ & $43^{\circ} 52^{\prime} 43^{\prime \prime}$ & 1.150 a 1.510 & Cwa & 117 & 40,48 & 1999 & 4,1 & 0,86 \\
\hline IN & Ingaí & 16,14 & $21^{\circ} 24^{\prime} 26^{\prime \prime}$ & $44^{\circ} 53^{\prime} 32^{\prime \prime}$ & 860 a 890 & $\mathrm{Cwb}$ & 103 & 29,29 & 2683 & 3,69 & 0,8 \\
\hline IT & Itutinga & 3,77 & $21^{\circ} 21^{\prime} 05^{\prime \prime}$ & $44^{\circ} 36^{\prime} 29^{\prime \prime}$ & 913 a 945 & $\mathrm{Cwb}$ & 123 & 27,37 & 2212,7 & 3,75 & 0,78 \\
\hline IU & Ibituruna & 59,75 & $21^{\circ} 10^{\prime} 00^{\prime \prime}$ & $44^{\circ} 50^{\prime} 25^{\prime \prime}$ & 820 a 980 & $\mathrm{Cwb}$ & 149 & 23,38 & 969,2 & 4,38 & 0,88 \\
\hline LA & Lafite & 25,95 & $21^{\circ} 12^{\prime} 58^{\prime \prime}$ & $44^{\circ} 48^{\prime} 08^{\prime \prime}$ & 825 a 863 & Cwb & 137 & 29,97 & 1610,7 & 4,2 & 0,86 \\
\hline LU & Luminárias & 77,91 & $21^{\circ} 29^{\prime} 11^{\prime \prime}$ & $44^{\circ} 55^{\prime} 20^{\prime \prime}$ & 880 a 1.000 & $\mathrm{Cwb}$ & 133 & 28,33 & 1830,5 & 4,19 & 0,86 \\
\hline LV & Lavras, UFLA & 5,72 & $21^{\circ} 24^{\prime} 42^{\prime \prime}$ & $44^{\circ} 58^{\prime} 10^{\prime \prime}$ & 918 a 937 & Cwb & 95 & 22,89 & 1291,1 & 3,61 & 0,79 \\
\hline MD & Madre de Deus & 20,66 & $21^{\circ} 29^{\prime} 03^{\prime \prime}$ & $44^{\circ} 22^{\prime} 32^{\prime \prime}$ & 915 a 980 & $\mathrm{Cwb}$ & 98 & 31,28 & 1144,2 & 3,69 & 0,81 \\
\hline MI & Mata da curva & 54,97 & $21^{\circ} 13^{\prime} 31^{\prime \prime}$ & $44^{\circ} 47^{\prime} 31^{\prime \prime}$ & 815 a 910 & $\mathrm{Cwb}$ & 114 & 23,64 & 1664 & 3,92 & 0,83 \\
\hline ML & Mata da Lagoa & 3,97 & $21^{\circ} 13^{\prime} 00^{\prime \prime}$ & $44^{\circ} 58^{\prime} 49^{\prime \prime}$ & 855 a 902 & $\mathrm{Cwb}$ & 133 & 29,14 & 1115,5 & 3,85 & 0,79 \\
\hline PB & Poço Bonito & 88,56 & $21^{\circ} 19^{\prime} 45^{\prime \prime}$ & $44^{\circ} 58^{\prime} 18^{\prime \prime}$ & 925 a 1.210 & Cwa & 151 & 22,93 & 1723,8 & 4,47 & 0,89 \\
\hline PI & Piedade & 24,95 & $21^{\circ} 29^{\prime} 16^{\prime \prime}$ & $44^{\circ} 06^{\prime} 02^{\prime \prime}$ & 1.040 a 1.150 & Cwa & 145 & 42,37 & 1481,7 & 4,3 & 0,86 \\
\hline $\mathrm{PN}$ & Pedra Negra & 72,34 & $21^{\circ} 05^{\prime} 25^{\prime \prime}$ & $44^{\circ} 56^{\prime} 52^{\prime \prime}$ & 835 a 885 & $\mathrm{Cwb}$ & 145 & 33,01 & 1677,3 & 4,1 & 0,82 \\
\hline $\mathrm{RM}$ & Rio das Mortes & 14,31 & $21^{\circ} 06^{\prime} 49^{\prime \prime}$ & $44^{\circ} 45^{\prime} 00^{\prime \prime}$ & 845 a 980 & $\mathrm{Cwb}$ & 139 & 25,09 & 2144 & 4,16 & 0,84 \\
\hline SE & Subestação & 8,73 & $21^{\circ} 13^{\prime} 17^{\prime \prime}$ & $44^{\circ} 57^{\prime} 47^{\prime \prime}$ & 910 a 940 & $\mathrm{Cwb}$ & 129 & 27,23 & 1500 & 4,13 & 0,85 \\
\hline SF & $\begin{array}{c}\text { São Francisco, } \\
\text { Varginha }\end{array}$ & 10 & $21^{\circ} 34^{\prime} 25^{\prime \prime}$ & $45^{\circ} 24^{\prime} 22^{\prime \prime}$ & 899 a 940 & $\mathrm{Cwb}$ & 111 & 23,65 & 1568 & 3,25 & 0,7 \\
\hline TD & Tiradentes & 271 & $21^{\circ} 03^{\prime} 55^{\prime \prime}$ & $44^{\circ} 05^{\prime} 58^{\prime \prime}$ & 920 a 1.340 & $\mathrm{Cwb}$ & 116 & 19,79 & 2386,7 & 4,24 & 0,87 \\
\hline
\end{tabular}

Cerne, Lavras, v. 18, n. 3, p. 361-370, jul./set. 2012 


\section{RESULTADOS E DISCUSSÃO}

\subsection{Composição florística}

No levantamento florístico, foram encontradas 111 espécies arbóreas pertencentes a 77 gêneros e 43 famílias. A família com maior número de espécies foi Myrtaceae com
18, seguida por Fabaceae com 15, Melastomataceae com 7, Lauraceae e Annonaceae com 5, Salicaceae, e Meliaceae com 4, Malpighiaceae, Rubiaceae e Sapindaceae com 3 espécies. Os gêneros com maior número de espécies foram Myrcia e Eugenia com 7 espécies cada, Miconia, com 6, Casearia com 4 e, Machaerium e Ocotea com 3 (Tabela 2).

Tabela 2 - Famílias, espécies amostradas e parâmetros estruturais da área de estudo. Col = número de coletor (Rafaela Pereira Naves); DA = Densidade Absoluta $\left(\right.$ ind.ha $\left.{ }^{-1}\right) ; \mathrm{DoA}=$ Dominância Absoluta $\left(\mathrm{m}^{2} \mathrm{ha}^{-1}\right) ; \mathrm{FA}=$ Frequência Absoluta $(\%)$; DR $=$ Densidade Relativa (\%); DoR = Dominância Relativa (\%); FR = Frequência Relativa (\%); VI= valor de importância; * = espécies amostradas fora das parcelas.

Table 2 - Families, species and structural parameters of the study area. Col = collector number (Rafaela Pereira Naves); DA = absolute density $\left(\right.$ ind, $\left.h \mathrm{~h}^{-1}\right) ; \mathrm{DoA}=$ absolute dominance $\left(\mathrm{m}^{2} h \mathrm{a}^{-1}\right) ; F A=$ absolute frequence $(\%) ; \mathrm{DR}=$ relative density $(\%) ; \mathrm{DoR}=$ relative dominance (\%); FR = relative frequence (\%),VI= importance value; $*=$ species sampled outside the plots.

\begin{tabular}{|c|c|c|c|c|c|c|c|c|c|}
\hline Família & Espécie & Col. & DA & DR & DoA & DoR & FA & FR & VI \\
\hline Achariaceae & Carpotroche brasiliensis (Raddi) Endl. & R57 & 1 & 0,06 & 0 & 0,01 & 0,04 & 0,23 & 0,3 \\
\hline Anacardiaceae & Tapirira obtusa (Benth.) J.D.Mitch. & R01 & 83 & 5,29 & 1,02 & 4,33 & 0,84 & 4,74 & 14,37 \\
\hline \multirow[t]{5}{*}{ Annonaceae } & Annona cacans Warm. & R67 & 7 & 0,45 & 0,09 & 0,37 & 0,12 & 0,68 & 1,5 \\
\hline & Annona sylvatica A.St.-Hil.* & R148 & & & & & & & \\
\hline & Duguetia lanceolata A.St.-Hil.* & R149 & & & & & & & \\
\hline & Guatteria sellowiana Schltdl. & $\mathrm{R} 130$ & 2 & 0,13 & 0,01 & 0,05 & 0,04 & 0,23 & 0,4 \\
\hline & Xylopia brasiliensis Spreng. & $\mathrm{R} 150$ & 18 & 1,15 & 0,15 & 0,62 & 0,24 & 1,35 & 3,12 \\
\hline Aquifoliaceae & Ilex cerasifolia Reissek & R48 & 5 & 0,32 & 0,05 & 0,22 & 0,2 & 1,13 & 1,66 \\
\hline \multirow[t]{2}{*}{ Araliaceae } & Dendropanax cuneatus (DC.) Decne. \& Planch. & R51 & 3 & 0,19 & 0,01 & 0,05 & 0,08 & 0,45 & 0,69 \\
\hline & Schefflera macrocarpa (Cham. \& Schltdl.) Frodin & R113 & 1 & 0,06 & 0,01 & 0,03 & 0,04 & 0,23 & 0,32 \\
\hline Arecaceae & Syagrus romanzoffiana (Cham.) Glassman & R47 & 5 & 0,32 & 0,08 & 0,33 & 0,2 & 1,13 & 1,78 \\
\hline \multirow[t]{2}{*}{ Asteraceae } & Piptocarpha macropoda (DC.) Baker & R07 & 3 & 0,19 & 0,1 & 0,43 & 0,04 & 0,23 & 0,85 \\
\hline & Vernonanthura divaricata (Spreng.) H.Rob. & R20 & 6 & 0,38 & 0,11 & 0,45 & 0,12 & 0,68 & 1,51 \\
\hline Bignoniaceae & Handroanthus ochraceus (Cham.) Mattos & R55 & 1 & 0,06 & 0,01 & 0,06 & 0,04 & 0,23 & 0,34 \\
\hline \multirow[t]{2}{*}{ Boraginaceae } & Cordia sellowiana Cham. & $\mathrm{R} 145$ & 1 & 0,06 & 0 & 0,01 & 0,04 & 0,23 & 0,3 \\
\hline & Cordia sp. $1^{*}$ & $\mathrm{R} 151$ & & & & & & & \\
\hline \multirow[t]{2}{*}{ Burseraceae } & Protium heptaphyllum (Aubl.) Marchand & $\mathrm{R} 152$ & 1 & 0,06 & 0,01 & 0,06 & 0,04 & 0,23 & 0,34 \\
\hline & Protium widgrenii Engl. & R33 & 33 & 2,1 & 0,31 & 1,3 & 0,52 & 2,93 & 6,34 \\
\hline Cannabaceae & Celtis pubescens (Kunth) Spreng. & R54 & 1 & 0,06 & 0,01 & 0,02 & 0,04 & 0,23 & 0,31 \\
\hline Crysobalanaceae & Couepia grandiflora (Mart. \& Zucc.) Benth. & R120 & 2 & 0,13 & 0,15 & 0,64 & 0,08 & 0,45 & 1,22 \\
\hline Cunoniaceae & Lamanonia ternata Vell. & R77 & 8 & 0,51 & 0,42 & 1,78 & 0,24 & 1,35 & 3,65 \\
\hline Cyatheaceae & Cyathea phalerata Mart. & R62 & 14 & 0,89 & 0,14 & 0,58 & 0,12 & 0,68 & 2,15 \\
\hline Erythroxylaceae & Erythroxylum suberosum A.St.-Hil. & $\mathrm{R} 146$ & 2 & 0,13 & 0,01 & 0,05 & 0,08 & 0,45 & 0,63 \\
\hline \multirow[t]{2}{*}{ Euphorbiaceae } & Alchornea triplinervia (Spreng.) Müll.Arg. & R63 & 1 & 0,06 & 0,02 & 0,07 & 0,04 & 0,23 & 0,36 \\
\hline & Croton floribundus Spreng. & $\mathrm{R} 101$ & 2 & 0,13 & 0,01 & 0,03 & 0,08 & 0,45 & 0,61 \\
\hline
\end{tabular}

Cerne, Lavras, v. 18, n. 3, p. 361-370, jul./set. 2012 
Tabela 2 - Continuação...

Table 2 - Continued...

\begin{tabular}{|c|c|c|c|c|c|c|c|c|c|}
\hline Família & Espécie & Col. & $\mathrm{DA}$ & DR & DoA & DoR & FA & FR & VI \\
\hline \multirow[t]{15}{*}{ Fabaceae } & Acosmium subelegans (Mohlenbr.) Yakovlev & R167 & 5 & 0,32 & 0,03 & 0,12 & 0,08 & 0,45 & 0,89 \\
\hline & Albizia polycephala (Benth.) Killip ex Record & R14 & 11 & 0,7 & 0,1 & 0,42 & 0,36 & 2,03 & 3,15 \\
\hline & Andira vermifuga (Mart.) Benth. & R04 & 2 & 0,13 & 0,24 & 1,03 & 0,08 & 0,45 & 1,6 \\
\hline & Bauhinia rufa (Bong.) Steud. & R89 & 1 & 0,06 & 0,01 & 0,03 & 0,04 & 0,23 & 0,32 \\
\hline & Bowdichia virgilioides Kunth & R90 & 6 & 0,38 & 0,07 & 0,28 & 0,24 & 1,35 & 2,02 \\
\hline & Copaifera langsdorffii Desf. & R38 & 135 & 8,61 & 3,18 & 13,54 & 0,64 & 3,61 & 25,76 \\
\hline & Dalbergia villosa (Benth.) Benth. & R35 & 7 & 0,45 & 0,15 & 0,62 & 0,2 & 1,13 & 2,19 \\
\hline & Machaerium hirtum (Vell.) Stellfeld & $\mathrm{R} 18$ & 1 & 0,06 & 0,01 & 0,03 & 0,04 & 0,23 & 0,32 \\
\hline & Machaerium stipitatum (DC.) Vogel & R87 & 1 & 0,06 & 0 & 0,01 & 0,04 & 0,23 & 0,3 \\
\hline & Machaerium villosum Vogel & R34 & 3 & 0,19 & 0,27 & 1,16 & 0,12 & 0,68 & 2,03 \\
\hline & Platycyamus regnellii Benth.* & R153 & & & & & & & \\
\hline & Platypodium elegans Vogel & R09 & 8 & 0,51 & 0,11 & 0,47 & 0,2 & 1,13 & 2,11 \\
\hline & Senna multijuga (Rich.) H.S.Irwin \& Barneby & R66 & 1 & 0,06 & 0,01 & 0,03 & 0,04 & 0,23 & 0,32 \\
\hline & Stryphnodendron adstringens (Mart.) Coville & R131 & 3 & 0,19 & 0,12 & 0,5 & 0,12 & 0,68 & 1,37 \\
\hline & $\begin{array}{l}\text { Tachigali rugosa (Mart. ex Benth.) Zarucchi \& } \\
\text { Pipoly }\end{array}$ & R06 & 33 & 2,1 & 4,21 & 17,93 & 0,44 & 2,48 & 22,52 \\
\hline Hypericaceae & Vismia brasiliensis Choisy. & R10 & 28 & 1,79 & 0,38 & 1,63 & 0,56 & 3,16 & 6,57 \\
\hline \multirow[t]{2}{*}{ Lamiaceae } & Aegiphila integrifolia (Jacq.) Moldenke & $\mathrm{R} 30$ & 1 & 0,06 & 0,01 & 0,02 & 0,04 & 0,23 & 0,31 \\
\hline & Vitex polygama Cham. & R132 & 1 & 0,06 & 0,01 & 0,03 & 0,04 & 0,23 & 0,32 \\
\hline \multirow[t]{5}{*}{ Lauraceae } & Cryptocarya arshesoniana $\mathrm{Mez}$ & R15 & 21 & 1,34 & 0,29 & 1,23 & 0,28 & 1,58 & 4,15 \\
\hline & Ocotea aciphylla (Nees \& Mart.) Mez & R19 & 1 & 0,06 & 0 & 0,01 & 0,04 & 0,23 & 0,3 \\
\hline & Ocotea corymbosa (Meisn.) Mez & R16 & 19 & 1,21 & 0,2 & 0,85 & 0,4 & 2,26 & 4,32 \\
\hline & Ocotea odorifera (Vell.) Rohwer & R17 & 4 & 0,26 & 0,02 & 0,1 & 0,12 & 0,68 & 1,03 \\
\hline & Persea rufotomentosa Nees. \& Mart. & R44 & 11 & 0,7 & 0,34 & 1,45 & 0,24 & 1,35 & 3,51 \\
\hline Magnoliaceae & Magnolia ovata (A.St.-Hil.) Spreng. & R59 & 12 & 0,77 & 0,29 & 1,24 & 0,12 & 0,68 & 2,69 \\
\hline \multirow[t]{3}{*}{ Malpighiaceae } & Byrsonima crassa Nied. & R05 & 2 & 0,13 & 0,03 & 0,14 & 0,08 & 0,45 & 0,72 \\
\hline & Byrsonima sericea DC. & R116 & 1 & 0,06 & 0 & 0,01 & 0,04 & 0,23 & 0,3 \\
\hline & Heteropterys byrsonimifolia A.Juss. & R100 & 10 & 0,64 & 0,11 & 0,47 & 0,16 & 0,9 & 2,01 \\
\hline \multirow[t]{2}{*}{ Malvaceae } & Luehea divaricata Mart. \& Zucc. & $\mathrm{R} 140$ & 1 & 0,06 & 0,02 & 0,09 & 0,04 & 0,23 & 0,38 \\
\hline & Luehea grandiflora Mart. \& Zucc. & R95 & 4 & 0,26 & 0,36 & 1,53 & 0,16 & 0,9 & 2,69 \\
\hline \multirow[t]{7}{*}{ Melastomataceae } & Miconia albicans (Sw.) Triana & R128 & 7 & 0,45 & 0,04 & 0,17 & 0,12 & 0,68 & 1,29 \\
\hline & Miconia chartacea Triana & R138 & 4 & 0,26 & 0,02 & 0,1 & 0,12 & 0,68 & 1,03 \\
\hline & Miconia pepericarpa DC. & $\mathrm{R} 75$ & 27 & 1,72 & 0,15 & 0,62 & 0,16 & 0,9 & 3,24 \\
\hline & Miconia tristis Spring & R46 & 1 & 0,06 & 0,02 & 0,08 & 0,04 & 0,23 & 0,37 \\
\hline & Miconia urophylla DC. & $\mathrm{R} 22$ & 11 & 0,7 & 0,09 & 0,37 & 0,16 & 0,9 & 1,97 \\
\hline & Miconia willdenowii Klotzsch ex Naudin & R137 & 11 & 0,7 & 0,24 & 1 & 0,28 & 1,58 & 3,29 \\
\hline & Trembleya parviflora (D.Don) Cogn. & R129 & 2 & 0,13 & 0,05 & 0,2 & 0,08 & 0,45 & 0,77 \\
\hline
\end{tabular}

Cerne, Lavras, v. 18, n. 3, p. 361-370, jul./set. 2012 
Tabela 2 - Continuação...

Table 2 - Continued...

\begin{tabular}{|c|c|c|c|c|c|c|c|c|c|}
\hline Família & Espécie & Col. & DA & DR & DoA & DoR & FA & FR & VI \\
\hline \multirow[t]{4}{*}{ Meliaceae } & Cabralea canjerana (Vell.) Mart. & R69 & 2 & 0,13 & 0,01 & 0,04 & 0,08 & 0,45 & 0,62 \\
\hline & Cedrela fissilis Vell.* & $\mathrm{R} 154$ & & & & & & & \\
\hline & Guarea guidonia (L.) Sleumer & $\mathrm{R} 72$ & 6 & 0,38 & 0,05 & 0,23 & 0,12 & 0,68 & 1,29 \\
\hline & Trichilia emarginata (Turcz.) C.DC. & R98 & 1 & 0,06 & 0 & 0,02 & 0,04 & 0,23 & 0,31 \\
\hline \multirow[t]{2}{*}{ Moraceae } & Ficus enormis Mart. ex Miq.* & R29 & & & & & & & \\
\hline & Ficus pertusa L.f. & $\mathrm{R} 40$ & 1 & 0,06 & 0 & 0,01 & 0,04 & 0,23 & 0,3 \\
\hline \multirow[t]{2}{*}{ Myrsinaceae } & Myrsine guianensis (Aubl.) Kuntze & R02 & 2 & 0,13 & 0,09 & 0,4 & 0,08 & 0,45 & 0,97 \\
\hline & Myrsine umbellata Mart. & $\mathrm{R} 28$ & 46 & 2,93 & 0,44 & 1,89 & 0,6 & 3,39 & 8,21 \\
\hline \multirow[t]{18}{*}{ Myrtaceae } & Blepharocalyx salicifolius (Kunth) O.Berg & R74 & 123 & 7,84 & 1,6 & 6,8 & 0,48 & 2,71 & 17,35 \\
\hline & Calyptranthes clusiifolia O.Berg & $\mathrm{R} 81$ & 11 & 0,7 & 0,05 & 0,2 & 0,28 & 1,58 & 2,48 \\
\hline & Calyptranthes widgreniana O.Berg & R142 & 1 & 0,06 & 0,01 & 0,03 & 0,04 & 0,23 & 0,32 \\
\hline & Eugenia aurata $\mathrm{O}$. Berg & $\mathrm{R} 112$ & 1 & 0,06 & 0,01 & 0,03 & 0,04 & 0,23 & 0,32 \\
\hline & Eugenia hiemailis Cambess. & R61 & 1 & 0,06 & 0 & 0,02 & 0,04 & 0,23 & 0,31 \\
\hline & Eugenia sonderiana O.Berg & R26 & 51 & 3,25 & 0,54 & 2,3 & 0,6 & 3,39 & 8,94 \\
\hline & Eugenia sp.1 & $\mathrm{R} 110$ & 1 & 0,06 & 0 & 0,02 & 0,04 & 0,23 & 0,31 \\
\hline & Eugenia sp.2 & R139 & 2 & 0,13 & 0,01 & 0,02 & 0,08 & 0,45 & 0,6 \\
\hline & Eugenia sp.3 & R166 & 1 & 0,06 & 0 & 0,02 & 0,04 & 0,23 & 0,31 \\
\hline & Eugenia uniflora L. & R32 & 66 & 4,21 & 0,45 & 1,89 & 0,64 & 3,61 & 9,71 \\
\hline & Marlieria sp. 1 & R106 & 1 & 0,06 & 0,01 & 0,03 & 0,04 & 0,23 & 0,32 \\
\hline & Myrcia hebepetala DC. & $\mathrm{R} 23$ & 3 & 0,19 & 0,07 & 0,29 & 0,08 & 0,45 & 0,93 \\
\hline & Myrcia oblongata DC. & $\mathrm{R} 24$ & 1 & 0,06 & 0,01 & 0,03 & 0,04 & 0,23 & 0,32 \\
\hline & Myrcia sp. & R123 & 1 & 0,06 & 0,01 & 0,03 & 0,04 & 0,23 & 0,32 \\
\hline & Myrcia splendens (Sw.) DC. & R08 & 194 & 12,37 & 1,36 & 5,79 & 0,88 & 4,97 & 23,13 \\
\hline & Myrcia tomentosa (Aubl.) DC. & R49 & 7 & 0,45 & 0,05 & 0,21 & 0,12 & 0,68 & 1,33 \\
\hline & Myrcia venulosa DC. & R41 & 1 & 0,06 & 0 & 0,01 & 0,04 & 0,23 & 0,3 \\
\hline & Myrcia vestita DC. & R136 & 1 & 0,06 & 0,01 & 0,03 & 0,04 & 0,23 & 0,32 \\
\hline Nyctagynaceae & Guapira hirsuta (Choisy) Lundell & R56 & 1 & 0,06 & 0 & 0,01 & 0,04 & 0,23 & 0,3 \\
\hline Peraceae & Pera glabrata (Schott) Poepp. ex Baill. & $\mathrm{R} 21$ & 79 & 5,04 & 1,12 & 4,74 & 0,68 & 3,84 & 13,62 \\
\hline Phyllanthaceae & Hyeronima alchorneoides Allemão & $\mathrm{R} 12$ & 1 & 0,06 & 0,01 & 0,03 & 0,04 & 0,23 & 0,32 \\
\hline Piperaceae & Piper gaudichaudianum Kunth* & R156 & & & & & & & \\
\hline Proteaceae & Roupala montana Aubl. & $\mathrm{R} 157$ & 1 & 0,06 & 0,05 & 0,19 & 0,04 & 0,23 & 0,48 \\
\hline \multirow[t]{3}{*}{ Rubiaceae } & Amaioua guianensis Aubl. & $\mathrm{R} 25$ & 88 & 5,61 & 0,59 & 2,51 & 0,6 & 3,39 & 11,5 \\
\hline & Cordiera concolor (Cham.) Kuntze & $\mathrm{R} 45$ & 2 & 0,13 & 0,03 & 0,11 & 0,04 & 0,23 & 0,46 \\
\hline & Faramea latifolia (Cham. \& Schltdl.) DC. & R141 & 1 & 0,06 & 0,01 & 0,03 & 0,04 & 0,23 & 0,32 \\
\hline Rutaceae & Zanthoxylum rhoifolium Lam. & R92 & 1 & 0,06 & 0,01 & 0,03 & 0,04 & 0,23 & 0,32 \\
\hline
\end{tabular}

Cerne, Lavras, v. 18, n. 3, p. 361-370, jul./set. 2012 
Tabela 2 - Continuação...

Table 2 - Continued..

\begin{tabular}{|c|c|c|c|c|c|c|c|c|c|}
\hline Família & Espécie & Col. & $\mathrm{DA}$ & DR & DoA & DoR & FA & FR & VI \\
\hline \multirow[t]{4}{*}{ Salicaceae } & Casearia arborea (Rich.) Urb. & R03 & 226 & 14,41 & 2,03 & 8,65 & 0,8 & 4,51 & 27,57 \\
\hline & Casearia lasiophylla Eichler & $\mathrm{R} 50$ & 4 & 0,26 & 0,02 & 0,1 & 0,08 & 0,45 & 0,8 \\
\hline & Casearia obliqua Spreng. & R76 & 1 & 0,06 & 0,04 & 0,15 & 0,04 & 0,23 & 0,44 \\
\hline & Casearia sylvestris $\mathrm{Sw}$. & R65 & 4 & 0,26 & 0,06 & 0,27 & 0,12 & 0,68 & 1,2 \\
\hline Sapotaceae & $\begin{array}{l}\text { Chrysophyllum marginatum (Hook. \& Arn.) } \\
\text { Radlk. }\end{array}$ & R107 & 1 & 0,06 & 0,01 & 0,04 & 0,04 & 0,23 & 0,33 \\
\hline \multirow[t]{3}{*}{ Sapindaceae } & Cupania vernalis Cambess.* & R158 & & & & & & & \\
\hline & Matayba juglandifolia (Cambess.) Radlk. & $\mathrm{R} 82$ & 1 & 0,06 & 0,01 & 0,03 & 0,04 & 0,23 & 0,32 \\
\hline & Matayba sp.1 & $\mathrm{R} 73$ & 1 & 0,06 & 0,02 & 0,06 & 0,04 & 0,23 & 0,35 \\
\hline \multirow[t]{2}{*}{ Siparunaceae } & Siparuna brasiliensis (Spreng.) A.DC.* & $\mathrm{R} 159$ & & & & & & & \\
\hline & Siparuna guianensis Aubl. & $\mathrm{R} 160$ & 1 & 0,06 & 0 & 0,01 & 0,04 & 0,23 & 0,3 \\
\hline Solanaceae & Solanum cernuum Vell. & $\mathrm{R} 78$ & 1 & 0,06 & 0 & 0,01 & 0,04 & 0,23 & 0,3 \\
\hline Urticaceae & Cecropia hololeuca Miq. & R71 & 2 & 0,13 & 0,05 & 0,23 & 0,08 & 0,45 & 0,81 \\
\hline Verbenaceae & Aloysia virgata (Ruiz \& Paiv.) A.Juss. & $\mathrm{R} 88$ & 3 & 0,19 & 0,03 & 0,14 & 0,08 & 0,45 & 0,79 \\
\hline \multirow[t]{2}{*}{ Vochysiaceae } & Callisthene major Mart. \& Zucc. & $\mathrm{R} 80$ & 5 & 0,32 & 0,28 & 1,18 & 0,16 & 0,9 & 2,4 \\
\hline & Vochysia tucanorum Mart. & R36 & 1 & 0,06 & 0,01 & 0,06 & 0,04 & 0,23 & 0,34 \\
\hline Indeterminadas & & & 22 & 1,4 & 0,65 & 2,77 & 0,88 & 4,97 & 9,14 \\
\hline
\end{tabular}

Comparando o perfil florístico do Parque São Francisco com o estudo de Oliveira-Filho e Fontes (2000) para as Florestas Semideciduais de Altitudes elevadas, é possível perceber que as famílias mais representativas do presente estudo também ocuparam as primeiras posições desse rank. Contudo, é importante ressaltar que a ordem de ocupação é diferente. Os autores citam que com aumento da altitude cresce a importância das famílias Asteraceae, Melastomataceae, Solanaceae e Myrsinaceae. Com exceção de Melastomataceae, as outras famílias não foram tão significativas no presente estudo.

Para gêneros com maior número de espécies, também houve divergências no rank. Com o aumento da altitude ocorre aumento dos gêneros Miconia, Mollinedia, Nectandra, Myrsine, Tibouchina, Solanum, Ilex, e Gomidesia (OLIVEIRA-FILHO; FONTES, 2000), com exceção de Miconia que apresentou muitas espécies, Myrsine, com duas e Ilex e Solanum, ambos com uma, os outros gêneros não ocorreram.

A partir dessa análise conclui-se que o dentro das florestas de altitudes elevadas $(>700 \mathrm{~m})$ o Parque São Francisco apresenta composição mais similar às florestas inferomontanas.

\subsection{Estrutura e diversidade da comunidade arbórea}

No levantamento estrutural, foram encontrados 1568 indivíduos.ha $^{-1}$ em 103 espécies, 83 gêneros e 41 famílias. A área basal total foi de $23,65 \mathrm{~m}^{2}$.ha- ${ }^{-1}$. O índice de diversidade de Shannon foi de 3,25 e a Equabilidade de Pielou 0,70.

O índice de diversidade de Shannon variou de 4.47 no Poço Bonito a 3.61 em Lavras, enquanto a equabilidade de Pielou variou de 0.89 no Poço Bonito a 0.78 em Itutinga.

Em comparação com outros remanescentes florestais da região do Alto Rio Grande, na Tabela 2, o que nos chama atenção é que o Parque São Francisco apresentou os menores valores de diversidade e equabilidade. Para a referida região, Pereira (2003) apontou alguns fatores que diminuem a diversidade dessas comunidades, como elevada proporção de bordas e severidade de impactos humanos. O Parque São Francisco, por estar situado em área urbana e pelo longo tempo de fragmentação, pode já estar sofrendo desses efeitos.

Esses valores baixos no contexto regional, particularmente para $J$, indicam uma concentração relativamente alta de abundâncias em um pequeno número de espécies que dominam a comunidade arbórea.

Cerne, Lavras, v. 18, n. 3, p. 361-370, jul./set. 2012 
Com relação a isso, as dez espécies de maior valor de importância somaram $58 \%$ do valor total e foram as seguintes: Casearia arborea, Copaifera langsdorffii, Myrcia splendens, Tachigali rugosa, Blepharocalyx salicifolius, Tapirira obtusa, Pera glabrata, Amaioua guianensis, Eugenia uniflora e Eugenia sonderiana, consideradas generalistas de habitat (OLIVEIRA-FILHO; FONTES, 2000) e amplamente distribuídas na região (PEREIRA, 2003).

\subsection{Análise do contexto florístico}

A DCA obteve para o eixo 1 o autovalor de $0,34 \mathrm{e}$ para o eixo 2 o autovalor de 0,13 (Figura 2). Segundo Braak (1995), valores acima de 0,5 indicam uma substituição de espécies ao longo dos eixos. O presente estudo indica gradientes curtos, ou seja, a maioria das espécies está distribuída por todo o gradiente e apenas uma minoria concentra-se em setores particulares. Isso significa que as áreas apresentam muitas espécies em comum.

Os eixos 1 e 2 sintetizaram cerca de $21 \%$ da variação total dos dados, sendo que valores baixos são

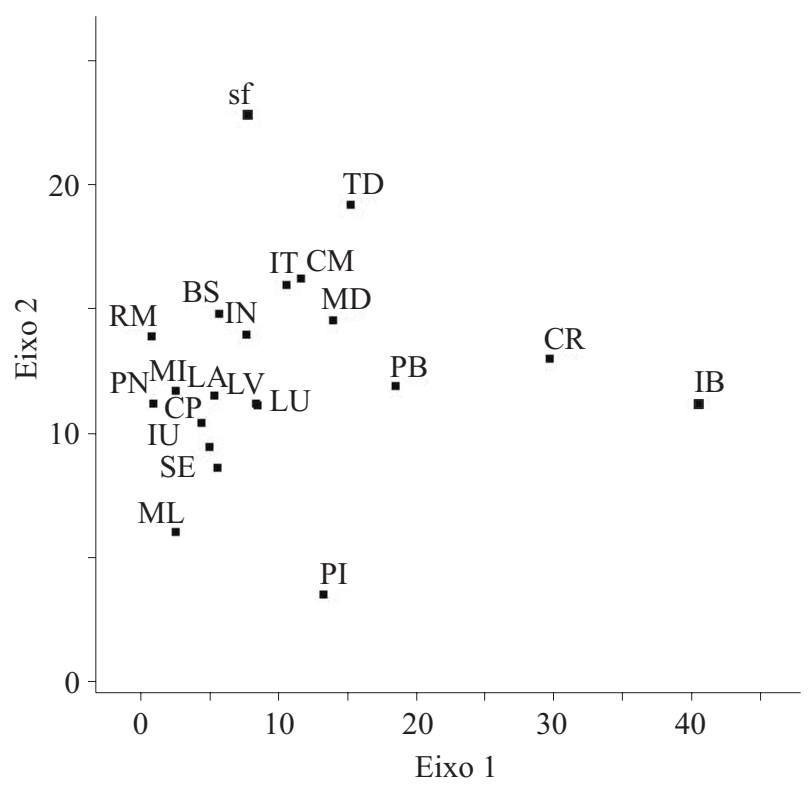

Figura 2 - Diagrama de ordenação dos dois primeiros eixos da análise de correspondência retificada referente aos dados florísticos dos 21 fragmentos da região do Alto Rio Grande, incluindo o Parque São Francisco. Para código de áreas ver Tabela 1.

Figure 2-Ordination diagram of first two axes of correspondence analysis of the floristic data of the 21 fragments of the Alto Rio Grande, including the Parque São Francisco. See Table 1. comuns em dados de vegetação, indicando uma elevada variação da distribuição das espécies não explicada pelos eixos (BRAAK, 1988).

A partir da observação do diagrama de ordenação das áreas, é possível perceber que o eixo 1 está ligado a variações de altitude. As áreas de maior altitude se encontraram do lado direito do diagrama (IB, CR, PB, TD), passando por áreas de altitude intermediária (como a do presente estudo) até as mais baixas (PN, RM, MI, ML, CP, LA).

Já é conhecido que a altitude é o fator que mais interfere na (dis) similaridade florística das florestas do Alto Rio Grande, agregando um complexo de variações climáticas (OLIVEIRA-FILHO et al., 1994c), sendo argumentado que o gradiente altitudinal também afeta a disponibilização de água (BERG; OLIVEIRA FILHO, 2000; GONZAGA et al., 2008; OLIVEIRA-FILHO; FONTES, 2000).

Algumas espécies típicas de florestas altomontanas são Hyptidendron asperrimum (Epling) Harley, Clethra scabra Pers., Croton organensis Baill., Drimys brasiliensis Miers, Weinmannia paulliniifolia Pohl, Meliosma sellowii Urb., Jacaranda subalpina W. Morawetz, Podocarpus sellowii Klotzsch e Araucaria angustifolia (Bert.) O. Kuntze (OLIVEIRA-FILHO; FONTES, 2000) todas não encontradas no Parque São Francisco. Enquanto Protium widgrenii Engl., Trichilia emarginata (Kurtz) C.DC., Vismia brasiliensis Choisy, Piptocarpha macropoda Baker e Calyptranthes clusiifolia O. Berg são mais tipicamente encontradas em altitudes intermediárias.

Outros aspectos que também interferem na distribuição de espécies é a presença de corpos d'água e a interface com outras formações florísticas, como cerrado (OLIVEIRA-FILHO; RATTER, 2002).

Segundo Berg e Oliveira-Filho (2000) a estacionalidade das chuvas e a influência dos corpos d'água, embora secundariamente, exercem papéis também importantes na distribuição das espécies. Enquanto padrões relacionados à fertilidade dos solos são mais difíceis de serem detectados em estudos de escalas mais amplas, como este, em razão da elevada heterogeneidade do substrato em níveis locais (GONZAGA et al., 2008).

Com base nos padrões florísticos apresentados entre o Parque São Francisco e as outras áreas do Alto Rio Grande, conclui-se que a área em questão encontrase dentro do que seria esperado para florestas estacionais semideciduais inferomentanas (OLIVEIRA-FILHO; FONTES, 2000).

Cerne, Lavras, v. 18, n. 3, p. 361-370, jul./set. 2012 


\section{REFERÊNCIAS}

APG III. An update of the Angiosperm Phylogeny Group classification for the orders and families of flowering plants: APG III. Botanical Journal of the Linnean Society, London, v. 161, p. 105-121, 2009.

BERG, E. van den; OLIVEIRA-FILHO, A. T. Composição florística e estrutura fitossociológica de uma floresta ripária em Itutinga, MG, e comparação com outras áreas. Revista Brasileira de Botânica, São Paulo, v. 23, n. 3, p. 231-253, 2000.

BRAAK, C. J. F. ter. CANOCO - A FORTRAN program for canonical community ordinationby (Partial) (Detrended) (Canonical) correspondence analysis and redundancy analysis. Version 2.1. Wageningen: Intitute of Applied Computer Science, 1988.

BRAAK, C. J. F. ter. Ordination. In: JOGMAN, R. H. G.; BRAAK, C. J. F. ter; TONGEREN, O. R. F. van. (Ed.). Data analysis in community and landscape ecology. Cambrigde: Cambrigde University, 1995. p. 91-173.

BROWER, J. E.; ZAR, J. H. Field and laboratory methods for general ecology. Dubuque: W. C. Brown, 1984. 226 p.

EITEN, G. Brazilian "Savannas". In: HUNTLEY, B. J.; WALKER, B. H. (Ed.). Ecology of tropical savannas. Berlin: Verlag, 1982. p. 25-47.

GONZAGA, A. P. D.; OLIVEIRA-FILHO, A. T.; MACHADO, E. L. M.; HARGREAVES, P.; MACHADO, J. N. M. Diagnóstico florístico-estrutural do componente arbóreo da floresta da Serra de São José, Tiradentes, MG, Brasil. Acta Botanica Brasilica, Porto Alegre, v. 22, p. 505520, 2008.

KENT, M.; COKER, P. Vegetation description and analysis, a pratical approach. London: Belhaven, 1992. 363 p.

MCCUNE, B.; MEFFORD, M. J. PC-ORD multivariate analysis of ecological data. Version 5.10. Gleneden Beach: MjM Software, 2006.

MITTERMEIER, R. A. Diversidade de primatas e a floresta tropical: estudos de casos do Brasil e Madagascar e a importância dos países com megadiversidade. In: WILSON,
O. E. (Ed.). Biodiversidade. Rio de Janeiro: Nova Fronteira, 1997. p. 186-200.

MUELLER-DOMBOIS, D.; ELLENBERG, H. Aims and methods of vegetation ecology. New York: Wiley, 1974. 574 p.

OLIVEIRA, A. R.; MARTINS, V. A. Projetos paisagísticos e arquitetônicos do Parque Florestal Municipal São Francisco de Assis, Varginha, MG. Belo Horizonte: IEF, 1986.

OLIVEIRA-FILHO, A. T.; FONTES, M. A. L. Patterns of floristic differentiation among Atlantic forests in southeastern Brazil, and the influence of climate. Biotropica, Washington, v. 31, p. 71-88, 2000.

OLIVEIRA-FILHO, A. T.; RATTER, J. A. Vegetation physiognomies and woody flora of the Cerrado Biome. In: PAULO, S.; OLIVEIRA, R. J. M. (Org.). The Cerrados of Brazil: ecology and natural history of a Neotropical savanna. New York: Columbia University, 2002. p. 91-120.

OLIVEIRA-FILHO, A. T.; SCOLFORO, J. R. S.; MELLO, J. M. Composição florística e estrutura comunitária de um remanescente de floresta semidecidua montana em Lavras, MG. Revista Brasileira de Botânica, São Paulo, v. 17, n. 2, p. 159-174, 1994a.

OLIVEIRA-FILHO, A. T.; VILELA, E. A.; CARVALHO, D. A.; GAVILANES, M. L. Effects of soils and topography on the distribution of tree species in a tropical riverine forest in south-eastern Brazil. Journal of Tropical Ecology, Cambridge, v. 10, p. 483-508, 1994b.

OLIVEIRA-FILHO, A. T.; VILELA, E. A.; GAVILANES, M. L.; CARVALHO, D. A. Comparison of the woody flora and soils of six areas of montane semideciduous forest in Southern Minas Gerais, Brazil. Edinburgh Journal of Botany, Edinburgh, v. 51, p. 355-389, 1994c.

PEREIRA, J. A. A. Efeitos dos impactos ambientais e da heterogeneidade ambiental sobre a diversidade e estrutura da comunidade arbórea de $\mathbf{2 0}$ fragmentos de florestas semidecíduas da região do Alto Rio Grande, Minas Gerias. 2003. 156 p. Tese (Doutorado em Ecologia, Conservação e Manejo da Vida Silvestre) - Universidade Federal de Minas Gerais, Belo Horizonte, 2003.

Cerne, Lavras, v. 18, n. 3, p. 361-370, jul./set. 2012 
RIBEIRO, M. C.; METZGER, J. P.; MARTENSEN, A. C.; PONZONI, F. J.; HIROTA, M. M. The Brazilian Atlantic Forest: how much is left, and how is the remaining forest distributed?: implications for conservation. Biological Conservation, Essex, v. 142, p. 1141-1153, 2009.

SILVA, V. F.; VENTURIN, N.; OLIVEIRA-FILHO, A. T.; CARVALHO, W. A. C.; BERG, E. van den; MACEDO, R.
L. G. Caracterização estrutural de um fragmento de floresta semidecídua no município de Ibituruna, MG. Cerne, Lavras, v. 9, n. 1, p. 95-110, 2003.

VELOSO, H. P.; RANGEL FILHO, A. L. R.; LIMA, J. C. A. Classificação da vegetação brasileira adaptada a um sistema universal. Rio de Janeiro: FIBGE, 1991. $123 \mathrm{p}$.

Cerne, Lavras, v. 18, n. 3, p. 361-370, jul./set. 2012 